Running Header: Conditions for Subscore Added Value Noninvariance

\title{
What are the Conditions Associated with Subscore Added Value Noninvariance? \\ Implications for Improving Subscore Interpretation Fairness
}

\author{
Joseph A. Rios \& Alejandra A. Miranda \\ University of Minnesota
}

\begin{abstract}
Author Note
All authors have departmental affiliation at the Department of Educational Psychology, University of Minnesota, Twin Cities.

This material is based upon the first author's dissertation, which was completed at the University of Massachusetts Amherst under the supervision of Stephen Sireci, Craig Wells, and Michael Lavine. Additional gratitude goes to Samuel Ihlenfeldt at the University of Minnesota for his willingness to provide feedback on the manuscript.

Author contribution statement: The first author conceived of the presented study, conducted the simulation analysis, wrote the manuscript, and conducted critical revisions to the manuscript. The second author was responsible for conducting the applied analyses.

Correspondence concerning this article should be addressed to Joseph Rios, University of Minnesota, 56 E River Rd, Minneapolis, MN 55455

Email: jrios@umn.edu
\end{abstract}

Citation:

Rios, J. A. \& *Miranda, A. A. (2020). What are the necessary conditions for the absence of subscore added value invariance? Implications for improving subscore interpretation fairness. Educational Measurement: Issues and Practice. Advance online publication. doi: 10.1111/emip.12379 


\begin{abstract}
Subscore added value analyses assume invariance across test taking populations; however, this assumption may be untenable in practice as differential subdomain relationships may be present among subgroups. The purpose of this simulation study was to understand the conditions associated with subscore added value noninvariance when manipulating: (a) subdomain test length, (b) differences in subgroup mean ability, and (c) subgroup differences in intersubdomain correlations. Results demonstrated that subscore added value was noninvariant for $24 \%$ to $100 \%$ of replications (depending on subdomain test length) when the subgroup difference in intersubdomain correlation was equal to .30 . To examine if this condition was met in practice, applied invariance analyses of three operational testing programs were conducted. Across these datasets, noninvariant subscore added value was present for some subdomains across sex and ethnic subgroups. Overall, these results indicate that subscore added value noninvariance is largely driven by differential intersubdomain correlations among subgroups, which may be present in some operational testing programs.

Keywords: Subscores, invariance, validity
\end{abstract}




\section{What are the Conditions Associated with Subscore Added Value Noninvariance? Implications for Improving Subscore Interpretation Fairness}

There has been increasing interest in providing stakeholders with subscores (i.e., scores on subtests) based on the assumption that such information may be a useful component for effective instruction, as it allows teachers to understand student learning challenges at a finegrained level and recognize their own accomplishments and deficiencies to enhance instructional competence (Firestone, 2014; Kunnan \& Jang, 2009). Although there has been limited research to examine this assumption, Muralidharan and Sundararaman (2010) found that, under the right conditions, providing subscore information may assist in improving student achievement by positively influencing teaching practices. While many stakeholders have increasingly demanded that subscores are reported (Brennan, 2012), the professional measurement community has stipulated that the distinctiveness and reliability from such scores should be demonstrated before they are given to stakeholders (Standard 1.14 of the Standards for Educational and Psychological Testing; American Educational Research Association, American Psychological Association, \& National Council on Measurement in Education, 2014).

Regardless of the increased demands for subscores by stakeholders, some individuals have concluded that without massive changes in test development, reporting subscores may not be a worthwhile endeavor (Wainer \& Feinberg, 2015). This assertion is based on numerous analyses of operational subscore utility, which have shown that, in practice, subscores are often lacking technical adequacy due to high intersubdomain correlations and/or low reliability (e.g., Haberman, 2008; Sinharay, 2010; Sinharay, Haberman, \& Puhan, 2007). One major reason for a lack of subdomain distinctiveness is that testing programs are often retrofitting subscores from essentially unidimensional assessments that were not designed specifically to provide 
information at the subtest-level (Luecht, Gierl, Tan, \& Huff, 2006). Furthermore, due to operational constraints (e.g., testing time), subtest lengths are often short. As an example, Goodman and Hambleton (2004) found some testing programs to report subscores based on as few as five items, while simulation studies have suggested that a minimum of 20 items are necessary for adequately reliable subscores (Feinberg, 2012; Sinharay, 2010). To address the issue of inadequate subscore reliability, researchers have proposed using collateral information (e.g., total score or performance on other subdomains; Wainer et al., 2001); however, these procedures have been shown to improve reliability at the cost of losing subdomain distinctiveness (Skorupski \& Carvajal, 2010).

\section{Subscore Added Value Invariance}

One of the major limitations of previous research evaluating the validity of subscore reporting is that such analyses dichotomously frame subscores as being either valid or invalid for all test takers without considering possible invariance of the test taker population. Such an assumption is made despite previous research having shown that there can be discrepant performance relationships between subdomains based on subgroup membership (see Stricker, 1993). To examine the tenability of this assumption in practice, Sinharay and Haberman (2014) investigated whether subscore added value (i.e., observed subscores are a better predictor of true subscores than the observed total score) was invariant for subgroups based on gender, ethnicity, and language across multiple testing contexts (e.g., language, teacher certification, and educational assessments). Their results showed that subscores for certain testing programs had diagnostic value for some test takers but not for others (i.e., subscore added value was noninvariant). These findings are of practical importance, as the latest federal education law, the Every Student Succeeds Act (ESSA; 2015), stipulates that every state is required to disaggregate 
achievement data (sample size permitting) for economically disadvantaged students, students from major racial and ethnic subgroups, and students with disabilities as well as limited English proficiency ${ }^{1}$. In the context of subscore reporting, disaggregating performance at a fine-grained level has the potential to inform educators and policy makers of areas for instructional improvement which could assist in reducing the achievement gap. However, before doing so, as demonstrated by the findings of Sinharay and Haberman (2014), it is imperative that evidence is collected to demonstrate added value invariance across subgroups (Standard 3.0; AERA, APA, \& NCME, 2014). A failure to do so may potentially lead to inaccurate comparative inferences by educational stakeholders.

\section{Study Objective}

To bring attention to this issue, this study builds upon the applied work of Sinharay and Haberman (2014) by systematically examining the factors that are associated with subscore added value noninvariance. Specifically, a simulation analysis was conducted that represented a context in which an assessment measuring two distinct but related skills is administered to a population that can be divided into two subgroups (e.g., language classification [English learner and non-English learner]) that may or may not differ in their underlying mean ability. In this context, the two subgroups are known to have differential intersubdomain correlations with one subgroup possessing a typically high intersubdomain correlation (hereon referred to the reference subgroup) and another possessing a lower one (hereon referred to as the focal subgroup). Based on this context, the following research questions are addressed:

\footnotetext{
${ }^{1}$ Additionally, it is common for states and school districts to disaggregate data for other subgroups, such as by gender and migratory status, to provide administrators with information on populations that may have a higher probability of failing academically or dropping out of school. For a review of student subgroups, the reader is referred to https://webcache.googleusercontent.com/search?q=cache:E_vYl_cWjWwJ:https://www.edglossary.org/studentsubgroup $/+\& \mathrm{~cd}=4 \& \mathrm{hl}=\mathrm{en} \& \mathrm{ct}=\mathrm{clnk} \& \mathrm{gl}=\mathrm{us}$
} 
How large of a difference in the intersubdomain correlation between subgroups is associated with subscore added value noninvariance when assuming equal subdomain reliabilities ${ }^{2}$ between subgroups? Furthermore, how does a difference in underlying subgroup mean ability moderate the relationship between differential intersubdomain correlations and subscore added value noninvariance?

The second objective of this study was to examine whether the conditions associated with noninvariant subscore added value found in the simulation analysis are present in operational testing contexts. Although Sinharay and Haberman (2014) examined operational data, this study investigates testing contexts that are unique from theirs and provides diversity in regards to the populations and constructs assessed. Findings from both the simulation and applied analyses have the potential to inform testing programs about the factors that may be associated with noninvariant subscores in practice.

\section{Simulation Study}

\section{Method}

The sections that follow describe the data generation process, conditions, and analyses that were employed for the simulation study.

Data generation. Data were generated separately for two subgroups administered an $n$ multiple-choice item test comprised of two subdomains (the total sample size was constrained to 40,000 simulees per subgroup; this same design was proposed by Sinharay and Haberman [2014]). First, the number of subdomains was constrained to two for simplicity's sake, as prior simulation research has demonstrated that the number of subdomains has no impact on subscore

\footnotetext{
${ }^{2}$ Subdomain reliabilities were constrained equal between subgroups based on generating data for an identical number of subdomain items with the same item parameters. These reliabilities were estimated from the generated data using coefficient alpha.
} 
added value (Sinharay, 2010). Second, this simulation context included only two subgroups, as the federally-mandated student classifications that states must present disaggregated data are most often dichotomized (e.g., students eligible for free and reduced lunch vs. students ineligible; students with disabilities vs. students without disabilities; students with limited English proficiency vs. students with adequate English proficiency). ${ }^{3}$ The two subgroups simulated in this study differed on the correlation underlying the ability estimates of the subdomains. Specifically, the reference subgroup was simulated to have subdomain scores with correlations that were constrained to .90 , to make the test highly unidimensional. This value was chosen as Sinharay (2010) found the median disattenuated intersubdomain correlation across 25 operational testing programs to be equal to .90 , thus, providing a typical scenario seen in practice. In contrast, the focal subgroup was simulated to have subscores with a range of intercorrelations that were investigated as an independent variable (more details are provided in the next section).

As the testing context simulated assumed a two-dimensional correlated-traits simple structure model, scores were generated by first sampling ability parameters from a multivariate normal distribution for simulees within each subgroup:

$$
\theta_{j} \sim N(\mu, \Sigma)
$$

where $\theta_{j}$ is a $2 \times 1$ vector of ability parameters, $\boldsymbol{\mu}$ is a $2 \times 1$ vector of mean ability values (for both subgroups, mean abilities were constrained equal across subdomains; however, differences in mean abilities were manipulated as a factor; more detail is provided below), and $\boldsymbol{\Sigma}$ is a $2 \times 2$

\footnotetext{
${ }^{3}$ The number of subgroups included would not be expected to change the inferences made from our simulation analyses as the approach examined compares a focal subgroup to a reference subgroup (akin to most invariance analyses). Therefore, if more than one focal subgroup were added, the same approach would be applied to the invariance analysis; however, there would be two separate comparisons for each focal subgroup compared to the reference subgroup.
} 
covariance matrix with the diagonal components equal to 1 and the off-diagonal components equal to the covariance of the intersubdomain abilities. Next, item responses were simulated separately for each subdomain using a unidimensional two-parameter logistic model:

$$
P\left(X_{i j}=1 \mid \theta_{j}, a_{i}, b_{i}\right)=\left(\frac{1}{1+\exp \left\{-1.7 a_{i}\left(\theta_{j}-b_{i}\right)\right\}}\right)
$$

where $P\left(X_{i j}=1 \mid \theta_{j}, a_{i}, b_{i}\right)$ is the probability of a correct response for simulee $j$ on item $i, \theta_{j}$ is simulee $j$ 's ability parameter for the subdomain of interest, $a_{i}$ is the slope parameter for item $i$, and $b_{i}$ is the difficulty parameter for item $i$. For each condition, item parameters were randomly sampled from a pool of 97 dichotomous items based on an operational administration of a unidimensional assessment of general learning outcomes. The mean discrimination and difficulty parameters of the item pool employed were $0.76(S D=0.29, \min =0.13, \max =1.67)$ and 0.21 $(S D=1.19, \min =-2.46, \max =3.81)$, respectively, indicating that data were generated for a moderately difficult and adequately discriminating assessment, assuming a standard normal ability distribution. As the underlying ability intercorrelations differed by subgroup, data generation was conducted separately for reference and focal subgroup simulees. This resulted in two $N_{g}$ x $I$ matrices for each replication, where $N_{g}$ is equal to 40,000 and $I$ is the total number of items (i.e., the stipulated subtest length x two subdomains).

Simulation conditions. In this study, three conditions were examined: (a) subtest length (three levels); (b) subgroup differences in mean ability (three levels); (c) subgroup differences in intersubdomain correlations (three levels). A fully-crossed design produced a total of 27 conditions. Each condition was replicated 100 times with a total of 80,000 simulees per replication (40,000 per subgroup; a total of 8,000,000 simulees across replications for each condition). 
Subtest length. Subtest length was included as an independent variable as it impacts estimates of both total test and subdomain reliability (coefficient alpha), which are known to impact subscore added value (see Sinharay, 2010). This study examined the following three subdomain test lengths (across subdomains, test length was held constant): (a) 10 items; (b) 20 items; (c) 40 items. The first level was chosen to represent a less than ideal situation in which the subdomain test length does not meet the minimal standard for adequate subdomain reliability as demonstrated by Sinharay (2010). However, as noted, in practice, such subdomain test lengths can be the norm as opposed to the exception (Goodman and Hambleton, 2004). The latter two subdomain test lengths have been previously examined in simulation studies of subscore added value (e.g., Sinharay, 2010) and represent sufficiently long subdomains for adequate subdomain reliability. The levels adopted in this simulation are well within what is to be expected in practice. This assertion is supported by Sinharay's (2010) analysis of 25 operational tests, which demonstrated average subdomain test lengths that ranged from 11 to 69 items.

Subgroup differences in mean ability. Subgroup mean ability differences were manipulated to investigate the role of subgroup impact (i.e., differences in subgroup mean ability) on subscore added value invariance. Though Sinharay and Haberman (2014) hypothesized that impact may not play an important role, their suggestion was limited based on inferences obtained from applied data. Therefore, to more systematically investigate this condition's influence, we examined subscore added value invariance under simulation of no impact (i.e., the two subgroups underlying mean ability was constrained equal to a standard normal distribution) and impact (i.e., the underlying mean abilities of the two subgroups differed). For the latter, two conditions were examined in which the mean ability between subgroups differed by 0.5 and $1 S D$. Specifically, the reference subgroup ability distribution was 
constrained to a standard normal distribution, while three distributions for the focal subgroup were manipulated: $N[0,1], N[-0.5,1]$, and $N[-1,1]$. The last two distributions assumed that the focal subgroup was on average of lower ability than the reference subgroup. This assumption and degree of difference have been examined in numerous studies that have investigated the role of subgroup impact on psychometric analyses (e.g., DeMars, 2010). Across all conditions, within each subgroup, mean ability was constrained equal across both subdomains.

Subgroup differences in intersubdomain correlations. This factor was examined as Sinharay and Haberman (2014) hypothesized that subscore added value noninvariance may likely be associated with differential intersubdomain correlations. To address this, three levels of intersubdomain correlations for the focal subgroup were evaluated: (a) .60; (b) .70; (c) .80. These three levels were chosen as they have been previously observed as disattenuated correlations in operational testing programs (e.g., Sinharay \& Haberman, 2014; those presented in the applied data analysis section of this paper). Thus, the differences in intersubdomain correlations examined between the reference and focal subgroups were $.10, .20$, and .30 .

Analysis of subscore added value. Haberman's (2008) procedure $^{4}$ was applied to evaluate whether subscores provide added value by assessing whether the observed subscore is a better predictor of the true subscore than the observed total score. This is done by evaluating the proportional reduction in mean squared error (PRMSE) for each predictor, which is conceptually similar to a reliability coefficient, ranging from 0 to 1 . The PRMSE for the observed subscore as

\footnotetext{
${ }^{4}$ Although Haberman and Sinharay (2012) developed a procedure to determine whether the inclusion of subgroup information (i.e., subgroup means and reliabilities) improves subscore estimation, they found that it led to no differences in subscore added value inferences across test taker demographic subgroups when compared to Haberman's (2008) method. In addition, Brennan (2012) as well as Feinberg and Wainer (2014a) have developed similar classical test theory approaches to evaluating individual-level subscore added value to Haberman (2008). As these authors have shown their procedures to lead to nearly identical subscore added value inferences as Haberman's (2008) procedure, the latter was employed in this study due to its popularity in the literature and in practice.
} 
a predictor of the true subscore $\left(\mathrm{PRMSE}_{\mathrm{s}}\right)$ has been shown to be equal to the subscore coefficient alpha (see Haberman, 2008 for more details). The PRMSE for the observed total score as a predictor of the true subscore $\left(\mathrm{PRMSE}_{\mathrm{x}}\right)$ is equal to:

$$
\rho^{2}\left(s_{t}, s_{x}\right) \rho^{2}\left(x_{t}, x\right)
$$

where $\rho^{2}\left(x_{t}, x\right)$ is the total test reliability, while $\rho^{2}\left(s_{t}, s_{x}\right)$ is computed as:

$$
\rho^{2}\left(s_{t}, s_{x}\right)=\frac{\left[\left(\operatorname{Cov}\left(s_{t}, x_{t}\right)\right]^{2}\right.}{\operatorname{Var}\left(s_{t}\right) \operatorname{Var}\left(x_{t}\right)}
$$

where $s_{t}$ and $x_{t}$ are the true subscore and total score, respectively (see Sinharay, Puhan, \& Haberman, 2011 for more details). Hence, a predictor with a larger PRMSE can provide more accurate diagnostic information than a predictor with a smaller PRMSE (i.e., if $\mathrm{PRMSE}_{\mathrm{s}}>$ PRMSE $_{x}$, then the observed subscore provides added value). Sinharay (2010) has shown via simulations that high levels of added value are associated with adequate subdomain test length (20 items or more) and intersubdomain correlations of .70 or less when using the criterion that $\mathrm{PRMSE}_{\mathrm{s}}$ must be greater than $\mathrm{PRMSE}_{\mathrm{x}}$ to allow for valid subscore reporting. However, research has suggested that such a criterion may be limited as the ultimate deciding factor in reporting subscores, particularly when considering the instability of PRMSE estimates for small sample sizes (Feinberg \& Wainer, 2015). To account for this limitation, the value-added ratio (VAR), $\mathrm{PRMSE}_{\mathrm{s}} / \mathrm{PRMSE}_{\mathrm{x}}$, has been proposed as a criterion for evaluating the reportability of subscores. Feinberg and Jurich (2017) have established VAR effect size guidelines, and have suggested that $\mathrm{VAR} \geq 1.1$ is a minimum requirement for meaningful subscore reporting.

In this study, subscore added value was calculated using the $R$ package subscore (version 3.1; Dai, Wang, \& Svetina, 2019). As the inferences concerning subscore added value are dependent on the accuracy of the PRMSE values, we considered the role of sampling error in our simulation analyses. Specifically, as shown by Feinberg and Wainer (2015), to confidently report 
PRMSE values to the second decimal place, one would need a sample size of approximately 40,000 test takers. To meet this requirement for PRMSE estimate stability, our sample size was constrained across all conditions to be equal to 40,000 simulees for each subgroup. For descriptive purposes, total test reliability, $\mathrm{PRMSE}_{\mathrm{s}}$ (subscore reliability), PRMSE $\mathrm{x}$, VAR, as well as added value decisions, were reported as an average across all subdomains and replications. The latter variable (added value decisions) was defined in two ways. First, we examined whether $\mathrm{PRMSE}_{\mathrm{s}}$ was greater than PRMSE $\mathrm{x}$ values following the approach outlined by Sinharay, Puhan, and Haberman (2011). Second, we utilized the VAR effect size guidelines proposed by Feinberg and Jurich (2017) to determine whether the added value present was of a large enough magnitude to provide meaningful subscore reporting. Upon determining added value for each subgroup separately, noninvariant subscore added value was calculated as the percentage of replications in which meaningful added value (indicated by VAR $\geq 1.1$ ) was present for the focal subgroup and not the reference subgroup (i.e., whether it would be worthwhile to report subscore performance for focal subgroup simulees and not reference subgroup simulees).

\section{Results}

Across all conditions, subscore added value invariance was found to be robust to subgroup differences in mean ability ${ }^{5}$. Therefore, the results presented below are based on the average results across this variable (see Table 1). As shown in Table 1, none of the reference subgroup conditions provided subscore added value for a typical correlation observed in practice (.90). Thus, subscore added value noninvariance required conditions in which added value was

\footnotetext{
${ }^{5}$ This factor was studied more extensively by adding conditions in which the focal subgroup intersubdomain correlation was constrained equal to that of the reference subgroup (.90) and both subdomain test length (10, 20, and 40 items) and subgroup mean ability differences (-0.5, and -1) varied. Across all six conditions (fully crossing subdomain test length and subgroup mean ability differences), there were $0 \%$ replications with noninvariant subscore added value. This result added further evidence for the robustness of subscore added value invariance to subgroup impact.
} 
obtained solely for the focal subgroup. Across conditions, we see that subscore added value was increasingly invariant as both the focal subgroup intersubdomain correlation increased and subdomain test length decreased. For example, when the intersubdomain correlation difference between subgroups was .10 and subdomain test lengths were 10 and 20 items, $0 \%$ of replications were found to be noninvariant. Maintaining the same intersubdomain correlation and increasing the subdomain test length to 40 items led to an absence of invariance for $100 \%$ of replications; however, upon closer examination, $0 \%$ of these replications were found to have a non-negligible effect size for the focal subgroup simulees, indicating minimal practical concern in there being differential subgroup added value.

As the subgroup difference in intersubdomain correlation increased to .20, the percentage of replications with subscore added value noninvariance was $50 \%, 65 \%$, and $100 \%$ for subdomain test lengths of 10, 20, and 40 items, respectively. Yet, across all subdomain test lengths, none of these replications exhibited a large enough effect size to indicate that the observed noninvariance was of practical importance. Finally, as expected, the largest degree of subscore added value noninvariance was observed for conditions in which the subgroup difference in intersubdomain correlation was equal to .30 . Specifically, $50 \%, 100 \%$, and $100 \%$ of replications were noninvariant for subdomain test lengths of 10, 20, and 40 items, respectively. In addition, many of these replications displayed non-negligible effect sizes, suggesting a real practical concern for the need to report subscore performance for focal subgroup simulees. For example, the percentage of replications that met the effect size criterion was $47 \%$ for a subdomain test length of 10 items and $24 \%$ for 20 items. This result suggested that although there were less focal subgroup replications with added value (PRMSEs $\left.>\mathrm{PRMSE}_{\mathrm{x}}\right)$ for the former condition, those that displayed added value possessed a higher rate of non-negligible 
effect sizes. One potential reason for this finding was that the average focal subgroup VAR for the condition with 20 items was 1.09 indicating that many of the replications were on the borderline of meeting the criterion. In contrast to these two subdmomain test lengths, $100 \%$ of replications for focal subgroup simulees met the effect size criterion for meaningful subscore reporting, indicating noninvariance of subscore added value, when each subdomain consisted of 40 items.

\section{Applied Analysis}

Results from the simulation analysis demonstrated that subscore added value invariance can be absent under certain conditions due to subgroup differences in subdomain intercorrelations. However, it is unclear whether these conditions are present in practice. To address this concern, data from multiple operational testing programs were analyzed for subscore added value invariance by identifiable subgroups.

\section{Data}

Data were obtained from three testing contexts. These testing contexts were selected as they are considerably different from each other in terms of their constructs, number of subdomains, subdomain test lengths, and intended populations. However, to maintain consistency with the simulation analysis, the demographic characteristics examined were all constrained to have two subgroups, while the number of subdomains were allowed to vary, as previous research has suggested that this factor does not impact subscore added value (Sinharay, 2010). Below is a description of each testing context.

National Intelligence Tests. The examined data come from 912 Estonian students from grades 6-8 administered the National Intelligence Tests in 2016. This test was originally developed in the 1930s by a team of psychologists (including E.L. Thorndike) to measure the 
intelligence of school children in grades $3-8$. It is a timed test administered in paper-and-pencil format, and consists of two scales (Scale A and B). For the purpose of this analysis, the subdomains investigated from Scale B were computation (requires addition, subtraction, multiplication, and division of both integers and fractions; 22 items), information (requires knowledge of everyday occurrences; e.g., "the day before Thursday is?"; 40 items), vocabulary (requires the knowledge of the qualities of different objects; 40 items), analogies (requires the transference of the relation of two given words to other presented words; 32 items), and comparisons (requires judging the sameness of sets of numbers, family names, and graphic symbols; 50 items). In addition to the total sample, added value was investigated for test takers based on self-identified sex (male $[n=413]$ and female $[n=499])$. For greater detail of this dataset, the reader is referred to Must and Must (2014).

Woodcock-Muñoz Language Survey-Reading (WLMS-R). Data were collected from 486 elementary school students (ranging in age from 5-9 years old) identified as English learners (ELs) who were administered the WLMS-R, which is a test that assesses English language proficiency in reading. It is comprised of two subtests - Letter Word Identification and Passage Comprehension (Woodcock, Muñoz-Sandovol, Ruef, \& Alvarado, 2005). The Letter Word Identification subtest (LWID) is comprised of 75 dichotomously-scored items and measures letter and word identification skills in English. The Passage Comprehension subtest (PCOMP) measures how well a student understands written discourse as it is being read in English and includes 33 dichotomously-scored items. During administration of this measure, the examinee is asked to silently read a short passage which contains a missing word. The examinee must then orally provide the missing word, ensuring that it makes sense within the context of the passage. Beginning items are based on symbolic learning and increase in difficulty. Subscore added value 
was evaluated for the total sample and by male $(n=228)$ and female $(n=258)$ subgroups. For validity evidence of this assessment, the reader is referred to Woodcock et al. (2005).

Big Five Inventory (BFI). The BFI is a 44 Likert (five-point) item multidimensional survey of personality which is comprised of five subdomains: extraversion (one's tendency to seek stimulation in the company of others; 8 items), agreeableness (one's tendency to be compassionate and cooperative towards others; 9 items), conscientiousness (one's tendency to show self-discipline, act dutifully, and aim for achievement; 9 items), neuroticism (one's vulnerability to unpleasant emotions; 8 items), and openness (one's degree of intellectual curiosity, creativity, and preference for novelty; 10 items; John, Naumann, \& Soto, 2008). Data for this analysis were collected from the administration of the BFI to 7,059 participants as part of the Understanding America Study (UAS), which is maintained by the Center for Economic and Social Research (CESR) at the University of Southern California. ${ }^{6}$ Participants were randomly selected using postal codes from a demographically diverse panel of adults nationwide aged 18 and over. For this test, subscore added value was evaluated for the total sample and by sex (male $[\mathrm{n}=3,079]$ and female $[\mathrm{n}=3,998]$ ) and ethnicity (White $[\mathrm{n}=6,013]$ and non-White $[\mathrm{n}=1,035]$ ). For validity evidence based on internal structure and relations to other variables for the BFI, the reader is referred to John et al. (2008).

\section{Analyses}

Subscore added value was evaluated using Haberman's (2008) method for the total and subgroup samples using the $R$ package subscore. Similar to the simulation analysis, subscore

\footnotetext{
${ }^{6}$ The content of this paper is solely the responsibility of the authors and does not necessarily represent the official views of USC or UAS.
} 
added value was determined based on the value-added ratio guidelines proposed by Feinberg and Jurich (2017).

\section{Results}

Results are presented separately for the National Intelligence Test, WMLS-R, and BFI. For each assessment, the subscore means, standard deviations, average disattenuated correlations, $\mathrm{PRMSE}_{\mathrm{s}}, \mathrm{PRMSE}_{\mathrm{x}}$, and value-added ratio values are presented for the total sample and subgroups examined (see Tables 2-4).

National Intelligence Tests. For the total sample $(\mathrm{N}=912)$, the test was found to have an overall reliability $(\alpha)$ equal to .92 , the subdomain reliabilities ranged from .56 to .87 , and the average disattenuated intersubdomain correlation for each subdomain ranged from .37 to .78 , suggesting a large degree of unique variance across subdomains (Table 2). The low intersubdomain correlations and adequate subdomain reliabilities (except for the computation subdomain $[\alpha=.56])$ were most likely the reason for all subdomains, except for the computation subdomain, meeting the value added ratio criterion for subscore added value. In addition to the total sample, Table 2 also presents results separately for female $(n=499)$ and male $(n=413)$ test takers. Comparing both subgroups, male test takers were found to have added value for all subdomains. This was not surprising as the subdomains with added value had reliabilities that ranged from .60 to .87 , a total test reliability of .91 , and intersubdomain correlations that ranged from .36 to .70 , indicating both reliable (except for the computation subdomain $[\alpha=.60]$ ) and distinct scores. Similar to males, the vocabulary, analogies, and comparisons subdomains were found to meet the criterion for subscore added value for females. These subdomains had reliabilities ranging from .73 to .86 and disattenuated correlations ranging from .29 to .62 . However, added value was not found for the computation and information subdomains. For the 
computation subdomain, this finding was most likely associated with a low subdomain reliability $(\alpha=.52)$, while for the information subdomain, the $\mathrm{PRMSE}_{\mathrm{s}}$ value was larger than that of the PRMSE , suggesting that added value was negligibly present, but not at a level that would be of practical importance to report. This finding illustrates noninvariant subscore added value by sex on National Intelligence Tests, Scale B, for the sample presented.

WLMS-R. Table 3 presents results for the total sample and by sex. Across samples, the reliabilities for the total test ( $\alpha$ ranged from .96 to .97) and both the letter word identification ( $\alpha$ ranged from .95 to .96 ) and passage comprehension ( $\alpha$ ranged from .88 to .91 ) subdomains were quite high. These reliabilities were found to be nearly identical between sex subgroups (see Table 3). The disattenuated intersubdomain correlation between the letter word identification and passage comprehension subdomains was equal to .79 for the total sample, while a difference was noted between females and males. Specifically, the disattenuated correlation for females was equal to .71 compared to .87 for males. In regard to total sample subscore added value, the value added ratio was greater than or equal to 1.1 for only the passage comprehension subdomain. A similar outcome was found for the female sample; however, in comparison, added value invariance was found to be absent for males as both subdomains did not meet the criterion for added value. This result was most likely associated with the higher intersubdomain correlation observed for this subgroup when compared to females.

Big Five Inventory. The results for the BFI are presented in Table 4 separately for the total sample and by sex and ethnicity. In regard to the total sample $(\mathrm{N}=7,094)$, subscore added value was found for all five subdomains. This was largely due to adequate total test reliability ( $\alpha$ $=.73$ ) and subdomain reliabilities ( $\alpha$ ranged from .75 to .82 ), as well as a wide range of disattenuated intersubdomain correlations ( $r$ ranged from -.12 to .82). Next, subscore added 
value invariance was examined by sex. Similar to the total sample, subscore added value was found for both sexes across all subdomains, as both subgroups possessed nearly identical total test (female: total test $\alpha=.72$; male: total test $\alpha=.73$ ) and subdomain (female: subdomain $\alpha$ ranged from .74 to .81; male: subdomain $\alpha$ ranged from .75 to .82) reliabilities as well as disattenuated intersubdomain correlations (female: $r$ ranged from - .10 to .87 ; male: $r$ ranged from -.20 to .76$)$.

Although subscore added value invariance was found by sex, noninvariance was present across ethnic subgroups. Specifically, similar to the total sample and sex subgroups, White testtakers possessed adequate total test $(\alpha=.73)$ and subdomain reliabilities ( $\alpha$ ranged from .75 to .82) with intersubdomain correlations that demonstrated unique variance across subdomains; however, non-White examinees were found to have differential subdomain reliabilities, particularly for the extraversion (non-White: $\alpha=.73$; White: $\alpha=.83$ ) and agreeableness (nonWhite: $\alpha=.86$; White: $\alpha=.75$ ) subdomains, and intersubdomain correlations ( $r$ ranged from .09 to 1 ; non-White: $\bar{r}=.61$; White: $\bar{r}=.33$ ) that were generally higher than those of White examinees. As a result, only the neuroticism subdomain met the VAR criterion, while all other subdomains did not display added value for non-White test-takers. This result indicated noninvariance of subscore added value for four of the five subdomains when comparing White and non-White individuals in this sample.

\section{Discussion}

The purpose of this study was to evaluate the conditions associated with subscore added value noninvariance and to determine whether these conditions were present in a small number of applied examples. Overall, the simulation results demonstrated that the noninvariance of subscore added value was robust to subgroup mean ability differences and was most highly 
associated with subgroup disparities in intersubdomain correlations. As an example, subscore added value invariance was maintained when the difference in subgroup intersubdomain correlation was as high as .20 , regardless of subdomain test length. However, when the intersubdomain subgroup difference increased to .30 , noninvariance was observed for $24 \%$ to $100 \%$ of replications, depending on subdomain test length. These results suggest that for meaningful noninvariance to be present, the degree of difference in intersubdomain correlations between or across subgroups must be relatively large. Though this may seem to be improbable in practice, our analysis of three applied datasets demonstrated meaningful subscore added value noninvariance for some subdomains and identifiable subgroups, which suggests that noninvariance may be an issue in certain operational contexts. Therefore, it is recommended that prior to reporting disaggregated subscore performance, practitioners should: (a) examine if the subgroups of interest possess differential subdomain reliabilities and/or intersubdomain correlations; (b) if so, conduct separate VAR analyses by subgroup to determine whether subscore added value invariance holds. By doing so, testing programs can strengthen the validity of inferences made from comparative subgroup interpretations.

\section{Limitations and Future Research}

Though a concerted effort was made to include the most pertinent independent variables along with respective levels in this study, it was impossible to include everything of importance. One area of research that was not covered in the present study was investigating the impact of the number of subdomain dimensions on subscore added value invariance. By changing the number of subdimensions, total test reliability would be expected to change from a classical test theory perspective; however, as noted by Sinharay (2008), such a difference in reliability has been found to not impact subscore added value inferences greatly. Additionally, this study 
constrained subdomain reliability to be equal across subgroups, however, as demonstrated in our applied examples, subgroups may possess small variations in reliability, which, when coupled with differences in intersubdomain correlations, may lead to subscore added value noninvariance. Therefore, it is recommended that future research investigates differential subdomain reliability across subgroups as an independent variable. A third limitation of this study was that multidimensional data were generated solely based on a simple structure model. As Feinberg and Wainer (2014b) recommended that complex items (i.e., an item loads on more than one dimension) increase intersubdomain correlations, it is anticipated that if an assessment possessed a complex test structure, the requirements necessary for subscore added value noninvariance would be even greater than shown in the conditions evaluated in this study. Fourth, this study solely employed Haberman's (2008) procedure as a criterion for assessing subscore added value. Although such an approach is popular in both research and practical contexts, it assumes that the model underlying the data is a multidimensional simple structure correlated-traits model. ${ }^{7}$ As a result, data for both subgroups were generated based on this model; however, in practice, it is possible that the underlying structure of multidimensional data may be represented with various models (e.g., higher-order or bifactor models). Hence, there is a need for further research to evaluate the robustness of inferences made when applying Haberman's (2008) procedure to multidimensional data that do not fit a correlated-traits model. Lastly, as with any analyses using applied data, the generalizability of the results is limited. As noted, only sex and ethnic differences in subscore added value were investigated with three datasets measuring intelligence, language, and personality. Therefore, it is recommended that

\footnotetext{
${ }^{7}$ The assumption also holds true for both Brennan's (2012) as well as Feinberg and Wainer's (2014a) approaches.
} 
future research examines more diverse subgroups ${ }^{8}$ and testing contexts to evaluate the presence of noninvariance in practice.

\section{Implications for Practice}

Regardless of these limitations, the results from this study illustrate that large differences in intersubdomain correlations can lead to noninvariant subscore added value, which may occur in some operational contexts. This has four implications for practice. First, the findings from this study and Sinharay and Haberman (2014) point to the need for operational testing programs to consistently evaluate the validity of reporting subdomains for all subgroups that data are to be disaggregated. By doing so, we may be able to limit the invalid inferences made by practitioners concerning subgroup differences in subdomain performance. Second, practitioners failing to incorporate an effect size measure similar to the one proposed by Feinberg and Jurich (2017) may incorrectly conclude that subscores are noninvariant. This was shown to be the case for a number of conditions in this study. Third, noninvariant subscore added value may point to possible intersubdomain correlation differences and/or differences in subtest difficulty among subgroups, which may have important implications for test development (Raymond \& Feinberg, 2015). As an example, it may indicate a need for test developers to review the balance of task topics and item types across subdomains as prior literature has shown there to be performance differences for these factors by subgroups (Federer, Nehm, \& Pearl, 2016; Livingston \& Rupp, 2004; Mazzeo, Schmitt, \& Bleistein, 1993). Fourth, the concept that subscores may be valid for some test takers elicits an important question, how should testing programs proceed? Does an

\footnotetext{
${ }^{8}$ One reviewer suggested examining subscore added value invariance for latent groups. However, as ESSA stipulates that data should be disaggregated for certain identifiable subgroups, we felt that an analysis based on latent groups would run contrary to the purpose of this study, which was to evaluate subscore reporting fairness in practice.
} 
absence of subscore added value invariance suggest that information should not be reported for the subdomain(s) of interest across all test takers? Is there an opportunity to report differential subscore information to stakeholders? As noted by Sinharay and Haberman (2014), evaluating added value invariance is only intended to verify the impact of reporting practices on examinee subgroups, akin to evaluating equating invariance. Thus, an absence of added value invariance should not motivate separate subscore reporting by subgroups. It is foreseeable that such a practice would lead to confusion among stakeholders and could raise further fairness questions not based on psychometric requirements (e.g., why is more detailed performance information reported for one ethnic subgroup and not another?). Thus, we would reiterate that added value noninvariance may provide important information for test development purposes. However, research that investigates how stakeholders utilize subscores to make inferences about test takers' performance is needed. As noted, this area of research has been extremely limited, even though debates in the literature have been long waged on technical considerations around subscores. By better understanding practitioner's viewpoints and coupling that with our evergrowing technical knowledge, we may be better able to serve teachers and students in improving learning. 


\section{References}

American Educational Research Association, American Psychological Association, \& National Council on Measurement in Education (2014). Standards for educational and psychological testing. Washington D.C.: American Educational Research Association.

Brennan, R. L. (2012). Utility indexes for decisions about subscores (CASMA Research Report 33). Iowa City, IA: Center for Advanced Studies in Measurement and Assessment.

Dai, S., Wang, X., \& Svetina, D. (2019). Subscore: Computing subscores in classical test theory and item response theory. R package version 3.1. Retrieved from https://cran.rproject.org/web/packages/subscore/subscore.pdf

DeMars, C. E. (2010). Type I error inflation for detecting DIF in the presence of impact. Educational and Psychological Measurement, 70, 961-972.

Every Student Succeeds Act of 2015, Pub. L. No. 114-95 § 114 Stat. 1177 (2015-2016).

Federer, M. R., Nehm, R. H., \& Pearl, D. K. (2016). Examining gender differences in written assessment tasks in biology: A case study of evolutionary explanations. $C B E-$ Life Sciences Education, 15, 1-11.

Feinberg, R. A. (2012). A simulation study of the situations in which reporting subscores can add value to licensure examinations (Unpublished doctoral dissertation). University of Delaware, Newark, DE.

Feinberg, R. A., \& Jurich, D. P. (2017). Guidelines for interpreting and reporting subscores. Educational Measurement: Issues and Practice, 36, 5-13.

Feinberg, R. A., \& Wainer, H. (2014a). A simple equation to predict a subscore's value. 
Educational Measurement: Issues and Practice, 33, 55-56.

Feinberg, R. A., \& Wainer, H. (2014b). When can we improve subscores by making them shorter?: The case against subscores with overlapping items. Educational Measurement: Issues and Practice, 33, 47-54.

Feinberg, R. A., \& Wainer, H. (2015). How much is enough? A reply to Sinharay, Haberman, \& Boughton. Educational Measurement: Issues and Practice, 34, 9.

Firestone, W.A. (2014). Teacher evaluation policy and conflicting theories of motivation. Educational Researcher, 43, 100-107.

Goodman, D. P., \& Hambleton, R. K. (2004). Student test score reports and interpretive guides: Review of current practices and suggestions for future research. Applied Measurement in Education, 17(2), 145-220.

Haberman, S. J. (2008). When can subscores have value? Journal of Educational and Behavioral Statistics, 33, 204-229.

Haberman, S. J., \& Sinharay, S. (2012). Does subgroup membership information lead to better estimation of true subscores? British Journal of Mathematical and Statistical Psychology, 66(3), 452-469.

John, O. P., Naumann, L. P., \& Soto, C. J. (2008). Paradigm shift to the integrative big-five trait taxonomy: History, measurement, and conceptual issues. In O. P. John, R. W. Robins, \& L. A. Pervin (Eds.), Handbook of personality: Theory and research (pp. 114158). New York, NY: Guilford Press.

Kunnan, A. J. and Jang, E. E. (2009). Diagnostic feedback in language assessment. In M. Long \& C. Doughty (Eds.), Handbook of second and foreign language teaching (pp. 610-625). Walden, MA: Wiley-Blackwell. 
Livingston, S. A., \& Rupp, S. L. (2004). Performance of men and women on multiple-choice and constructed-response tests for beginning teachers (RR-04-48). Educational Testing Service: Princeton, NJ.

Luecht, R. M., Gierl, M. J., Tan, X., \& Huff, K. (April, 2006). Scalability and the Development of Useful Diagnostic Scales. Paper presented at the Annual Meeting of the National Council on Measurement in Education, San Francisco, CA.

Mazzeo, J., Schmitt, A. P., \& Bleistein, C. A. (1993). Sex-related performance differences on constructed-response and multiple-choice sections of advanced placement examinations (College Board Report No. 92-7, ETS Research Report No. 93-5). College Entrance Examination Board, New York, NY.

Muralidharan, K., \& Sundararaman, V. (2010). The impact of diagnostic feedback to teachers on student learning: Experimental evidence from India. The Economic Journal, 120, F187-F203.

Must, O., \& Must, A. (2014). Data from "Changes in test-taking patterns over time” concerning the Flynn Effect in Estonia. Journal of Open Psychology Data, 2, e2. DOI: http://dx.doi.org/10.5334/jopd.ab

Raymond, M., \& Feinberg, R. (2015, April). Subscores aren't for everyone: Alternative strategies for evaluating subscore utility. Paper presented at the annual conference of the National Council on Measurement in Education, Chicago, IL.

Sinharay, S. (2010). How often do subscores have added value? Results from operational and simulated data. Journal of Educational Measurement, 47, 150-174.

Sinharay, S., \& Haberman, S. J. (2014). An empirical investigation of population invariance in the value of subscores. International Journal of Testing, 14(1), 22-48. 
Sinharay, S., Haberman, S., \& Puhan, G. (2007). Subscores based on classical test theory: To report or not to report. Educational Measurement: Issues and Practice, 26, 21-28. Sinharay, S., Puhan, G., \& Haberman, S. J. (2011). An NCME instructional module on subscores. Educational Measurement: Issues and Practice, 30(3), 29-40.

Skorupski, W. P., \& Carvajal, J. (2010). A comparison of approaches for improving the reliability of objective level scores. Educational and Psychological Measurement, 70, $357-375$.

Stricker, L. J. (1993). Discrepant LSAT subscores (Technical Report No. 93-01). Newtown, PA: Law School Admission Council.

Wainer, H., \& Feinberg, R. (2015). For want of a nail: Why unnecessarily long tests may be impeding the progress of Western civilisation. Significance, 12, 16-21.

Wainer, H., Vevea, J.L., Camacho, F., Reeve, B.B., Rosa, K., Nelson, L., et al. (2001). Augmented scores: "Borrowing Strength" to compute scores based on small numbers of items. In D. Thissen \& H. Wainer (Eds.), Test scoring (pp. 343-387). Mahwah, NJ: Lawrence Erlbaum Associates. 
Table 1

Subscore added value invariance results as a function of subdomain test length and intersubdomain correlation difference

\begin{tabular}{|c|c|c|c|c|c|c|c|c|c|}
\hline \multirow[b]{2}{*}{ Subdomain Test Length } & \multirow[b]{2}{*}{$\begin{array}{l}\text { Correlation } \\
\text { Difference }\end{array}$} & \multicolumn{4}{|c|}{ Reference Subgroup } & \multicolumn{4}{|c|}{ Focal Subgroup } \\
\hline & & Total $\alpha$ & $\mathrm{PRMSE}_{\mathrm{s}}$ & $\mathrm{PRMSE}_{\mathrm{x}}$ & VAR & Total $\alpha$ & $\mathrm{PRMSE}_{\mathrm{s}}$ & $\mathrm{PRMSE}_{\mathrm{x}}$ & VAR \\
\hline \multirow[t]{3}{*}{10} & .10 & .63 & .48 & .61 & 0.78 & .62 & .48 & .57 & 0.84 \\
\hline & .20 & .63 & .48 & .61 & 0.78 & .61 & .48 & .52 & 0.91 \\
\hline & .30 & .63 & .48 & .61 & 0.78 & .59 & .48 & .48 & 0.99 \\
\hline \multirow[t]{3}{*}{20} & .10 & .79 & .67 & .76 & 0.88 & .78 & .67 & .71 & 0.94 \\
\hline & .20 & .79 & .67 & .76 & 0.88 & .77 & .67 & .66 & 1.01 \\
\hline & .30 & .79 & .67 & .76 & 0.88 & .76 & .67 & .61 & 1.09 \\
\hline \multirow[t]{3}{*}{40} & .10 & .89 & .81 & .85 & 0.96 & .88 & .81 & .80 & 1.02 \\
\hline & .20 & .89 & .81 & .85 & 0.96 & .88 & .81 & .75 & 1.08 \\
\hline & .30 & .89 & .81 & .85 & 0.96 & .87 & .81 & .70 & 1.16 \\
\hline
\end{tabular}

Note. The results displayed here are taken as an average across both subdomains and conditions of subgroup mean ability differences. 
Table 2

Subscore Added Value by Sex for the National Intelligence Tests

\begin{tabular}{|c|c|c|c|c|c|c|c|c|}
\hline Subscore & $\begin{array}{l}\text { No. of } \\
\text { Items }\end{array}$ & Mean & SD & $\bar{r}_{d i s}$ & $\mathrm{PRMSE}_{\mathrm{s}}$ & PRMSE $_{x}$ & VAR & $\begin{array}{c}\text { Subscore } \\
\text { Added } \\
\text { Value? } \\
(\text { VAR } \geq 1.1)\end{array}$ \\
\hline & \multicolumn{8}{|c|}{ Total Sample $(n=912)$} \\
\hline Computation & 22 & 10.93 & 2.29 & .57 & .56 & .54 & 1.03 & No \\
\hline Information & 40 & 22.08 & 4.95 & .61 & .75 & .65 & 1.15 & Yes \\
\hline Vocabulary & 40 & 31.95 & 4.15 & .58 & .73 & .62 & 1.18 & Yes \\
\hline Analogies & 32 & 19.98 & 5.81 & .56 & .86 & .63 & 1.37 & Yes \\
\hline \multirow[t]{2}{*}{ Comparisons } & 50 & 38.47 & 5.96 & .43 & .87 & .47 & 1.85 & Yes \\
\hline & \multicolumn{8}{|c|}{$\begin{array}{c}\text { Sex Subgroups } \\
\text { Females }(\mathrm{n}=499)\end{array}$} \\
\hline Computation & 22 & 11.06 & 2.19 & .59 & .52 & .56 & 0.93 & No \\
\hline Information & 40 & 21.99 & 4.85 & .65 & .74 & .72 & 1.03 & No* \\
\hline Vocabulary & 40 & 32.10 & 4.11 & .61 & .73 & .66 & 1.11 & Yes \\
\hline Analogies & 32 & 21.08 & 5.61 & .54 & .85 & .60 & 1.42 & Yes \\
\hline \multirow[t]{2}{*}{ Comparisons } & 50 & 39.36 & 5.48 & .43 & .86 & .43 & 2.00 & Yes \\
\hline & \multicolumn{8}{|c|}{ Males $(n=413)$} \\
\hline Computation & 22 & 10.76 & 2.40 & .55 & .60 & .52 & 1.15 & Yes* \\
\hline Information & 40 & 22.19 & 5.07 & .58 & .76 & .61 & 1.25 & Yes \\
\hline Vocabulary & 40 & 31.78 & 4.20 & .55 & .73 & .57 & 1.28 & Yes \\
\hline Analogies & 32 & 18.66 & 5.77 & .56 & .85 & .65 & 1.31 & Yes \\
\hline Comparisons & 50 & 37.39 & 6.34 & .42 & .87 & .50 & 1.74 & Yes \\
\hline
\end{tabular}

Note. $\bar{r}_{\text {dis }}$ represents the average disattenuated correlation across subdomains. Total score reliability for all, female, and male test takers was $.92, .92, .91$, respectively. *Denotes that the subscore added value decision differs from the total sample. 
Table 3

Subscore Added Value by Sex for the Woodcock Muñoz Language Survey Revised (WMLS-R)

Subscore

$\begin{array}{lclllll}\text { Subscore } & \begin{array}{c}\text { No. of } \\ \text { Items }\end{array} \text { Mean } & \text { SD } & \bar{r}_{\text {dis }} & \text { PRMSEs } & \text { PRMSEx } & \text { VAR Added Value? }\end{array}$

$(\mathrm{VAR} \geq 1.1)$

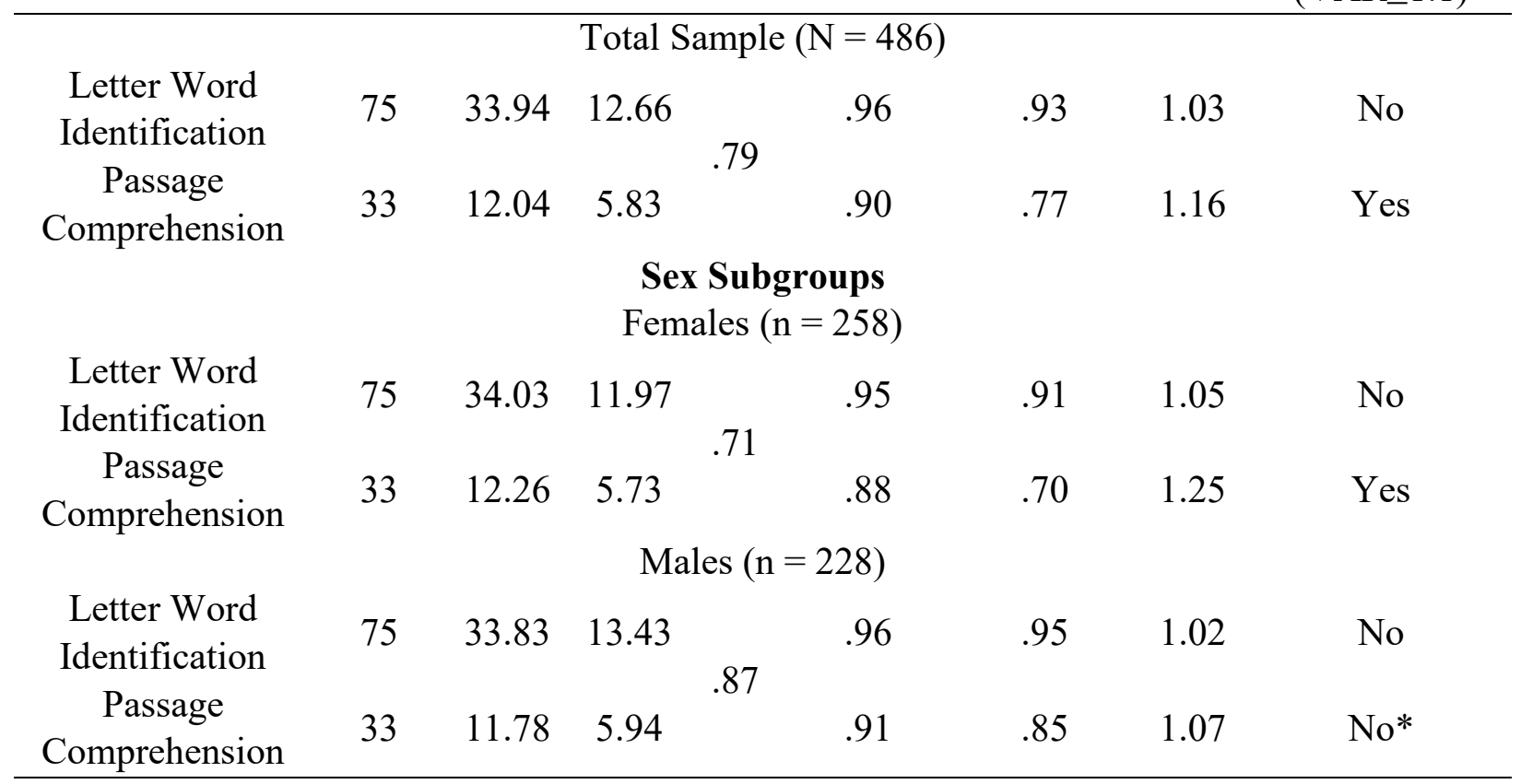

Note. $\bar{r}_{\text {dis }}$ represents the average disattenuated correlation across subdomains. Total score reliability for all, female, and male was $.97, .96, .97$, respectively. *Denotes that the subscore added value decision differs from the total sample. 
Table 4

Subscore Added Value by Sex and Ethnicity for the Big Five Inventory (BFI)

\begin{tabular}{|c|c|c|c|c|c|c|c|c|}
\hline Subscore & $\begin{array}{c}\text { No. } \\
\text { of } \\
\text { Items }\end{array}$ & Mean & SD & $\bar{r}_{d i s}$ & $\mathrm{PRMSE}_{\mathrm{S}}$ & PRMSEX $_{X}$ & VAR & $\begin{array}{c}\text { Subscore } \\
\text { Added Value? } \\
(\mathrm{VAR} \geq 1.1)\end{array}$ \\
\hline \multicolumn{9}{|c|}{ Total Sample $(\mathrm{N}=7,094)$} \\
\hline Extraversion & 8 & 26.18 & 7.03 & .40 & .81 & .51 & 1.60 & Yes \\
\hline Agreeableness & 9 & 35.75 & 6.85 & .48 & .75 & .61 & 1.23 & Yes \\
\hline Conscientiousness & 9 & 35.85 & 6.93 & .48 & .76 & .62 & 1.23 & Yes \\
\hline Neuroticism & 8 & 20.88 & 6.89 & -.06 & .82 & .04 & 18.86 & Yes \\
\hline Openness & 10 & 35.79 & 7.66 & .49 & .77 & .66 & 1.18 & Yes \\
\hline \multicolumn{5}{|c|}{ Sex Subgroups } & \multicolumn{4}{|c|}{ Females $(\mathrm{n}=3,998)$} \\
\hline Extraversion & 8 & 26.32 & 7.17 & .43 & .81 & .53 & 1.54 & Yes \\
\hline Agreeableness & 9 & 36.33 & 6.96 & .52 & .74 & .64 & 1.15 & Yes \\
\hline Conscientiousness & 9 & 35.92 & 7.04 & .52 & .75 & .65 & 1.15 & Yes \\
\hline Neuroticism & 8 & 21.65 & 7.04 & -.03 & .81 & .06 & 14.16 & Yes \\
\hline Openness & 10 & 35.30 & 7.88 & .53 & .77 & .68 & 1.13 & Yes \\
\hline \multicolumn{9}{|c|}{ Males $(n=3,079)$} \\
\hline Extraversion & 8 & 26.01 & 6.84 & .36 & .82 & .48 & 1.72 & Yes \\
\hline Agreeableness & 9 & 35.02 & 6.60 & .42 & .75 & .56 & 1.35 & Yes \\
\hline Conscientiousness & 9 & 35.78 & 6.77 & .43 & .78 & .58 & 1.35 & Yes \\
\hline Neuroticism & 8 & 19.88 & 6.57 & -.12 & .82 & .02 & 40.58 & Yes \\
\hline Openness & 10 & 36.43 & 7.31 & .45 & .78 & .64 & 1.21 & Yes \\
\hline \multicolumn{9}{|c|}{$\begin{array}{l}\text { Ethnic Subgroups } \\
\text { White }(\mathrm{n}=6,013)\end{array}$} \\
\hline Extraversion & 8 & 26.23 & 6.96 & .35 & .83 & .47 & 1.75 & Yes \\
\hline Agreeableness & 9 & 35.83 & 6.54 & .40 & .75 & .54 & 1.39 & Yes \\
\hline Conscientiousness & 9 & 35.98 & 6.60 & .42 & .77 & .56 & 1.36 & Yes \\
\hline Neuroticism & 8 & 21.17 & 6.81 & -.12 & .82 & .03 & 31.25 & Yes \\
\hline Openness & 10 & 35.93 & 7.38 & .42 & .78 & .60 & 1.30 & Yes \\
\hline \multicolumn{9}{|c|}{ Non-White $(\mathrm{n}=1,035)$} \\
\hline Extraversion & 8 & 25.85 & 7.43 & .69 & .73 & .73 & .99 & No* \\
\hline Agreeableness & 9 & 35.28 & 8.40 & .73 & .72 & .86 & .84 & No* \\
\hline Conscientiousness & 9 & 35.05 & 8.58 & .71 & .76 & .82 & .92 & No* \\
\hline Neuroticism & 8 & 19.30 & 7.14 & .17 & .79 & .13 & 6.08 & Yes \\
\hline Openness & 10 & 34.96 & 9.06 & .77 & .74 & .88 & .85 & No* \\
\hline
\end{tabular}

Note. $\bar{r}_{\text {dis }}$ represents the average disattenuated correlation across subdomains. Total score reliability for all, female, male, White, and Non Whites was .73, .72, .73, .73, .73, respectively. *Denotes that the subscore added value decision differs from the total sample. For this measure, very large VAR values were observed for subdomains that had disattenuated correlations that were near zero. 